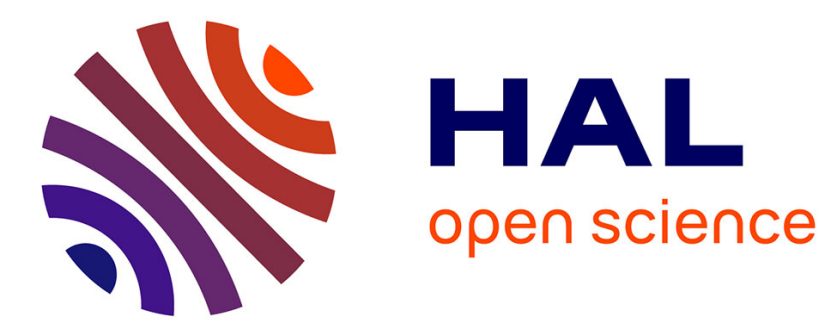

\title{
Undulation instability under shear in smectic A liquid crystals
}

\author{
P. Oswald, S.I. Ben-Abraham
}

\section{To cite this version:}

P. Oswald, S.I. Ben-Abraham. Undulation instability under shear in smectic A liquid crystals. Journal de Physique, 1982, 43 (8), pp.1193-1197. 10.1051/jphys:019820043080119300 . jpa-00209496

\section{HAL Id: jpa-00209496 https://hal.science/jpa-00209496}

Submitted on 1 Jan 1982

HAL is a multi-disciplinary open access archive for the deposit and dissemination of scientific research documents, whether they are published or not. The documents may come from teaching and research institutions in France or abroad, or from public or private research centers.
L'archive ouverte pluridisciplinaire HAL, est destinée au dépôt et à la diffusion de documents scientifiques de niveau recherche, publiés ou non, émanant des établissements d'enseignement et de recherche français ou étrangers, des laboratoires publics ou privés. 
Classification

Physics Abstracts

$47.15-61.30$

\title{
Undulation instability under shear in smectic A liquid crystals
}

\author{
P. Oswald and S. I. Ben-Abraham (*) \\ Laboratoire de Physique des Solides, Bât. 510, Université de Paris-Sud, 91405 Orsay Cedex, France
}

(Reçu le 25 février 1982, accepté le 15 avril 1982)

\begin{abstract}
Résumé. - Nous étudions l'instabilité d'ondulation dans un smectique A sous cisaillement parallèle aux couches. Nous cherchons la dilatation au seuil d'apparition d'une ondulation dont le vecteur d'onde fait un angle $\theta$ avec la direction de l'écoulement. Nous trouvons une relation intégrale qui montre que la dilatation est nécessairement positive. Nous développons une méthode de perturbations pour calculer la dilatation explicitement en fonction de l'angle $\theta$ et du taux de cisaillement. Nous montrons qu'une ondulation de vecteur d'onde perpendiculaire à la direction de cisaillement n'est pas affectée par l'écoulement et apparaît pour un seuil de dilatation minimum et égal à la valeur statique. Nous concluons qu'une texture rectangulaire se développe pour des dilatations plus grandes en accord avec le réseau de domaines focaux observé expérimentalement.
\end{abstract}

Abstract. - We study the undulation instability in a smectic A in the presence of shear flow parallel to the layers. We look for the dilation at the threshold where an undulation appears with a wave vector inclined at an angle $\theta$ to the direction of flow. We find an integral relation showing that the dilation is necessarily positive. We develop a perturbation method to calculate the dilation explicitly as a function of the angle $\theta$ and the shear rate. We show that an undulation with wave vector perpendicular to the shear direction is not affected by the flow and has a minimum threshold dilation equal to the static value. We conclude that a rectangular texture develops at higher dilations in agreement with the rectangular focal domain pattern observed experimentally.

Introduction - We deal with a smectic A liquid crystal subjected to a dilative strain perpendicular to the layers. Above a certain threshold value of the dilation, the layers develop a static undulation with some wave vector $\mathbf{q}$ of arbitrary orientation within a plane parallel to the layers $[1,2,3,4]$.

We want to ask and find an answer to the question : what happens to the undulation and how is the threshold modified when the sample is dilated while being simultaneously sheared parallel to the layers.

1. Formulation of the problem. - Consider a smectic sample of thickness $2 d$ sheared betwen two plane parallel plate moving at equal and opposite velocities $\pm \mathbf{V}_{0}$. Homeotropic anchoring keeps the smectic layers parallel to the plates (Fig. 1). The flow is described by the equation of Navier-Stokes [5] :

$$
\rho \frac{\mathrm{d} \mathbf{V}}{\mathrm{d} t}=\eta \nabla^{2} \mathbf{V}-\operatorname{grad} P+\mathbf{G}
$$

where $\mathbf{V}$ is the velocity field, $P$ the pressure, and $\mathbf{G}=(0,0, G)$ is a volume force density due to the

(*) Permanent address : Department of Physics, BenGurion University of the Negev, 84105 Beer-Sheva, Israel.

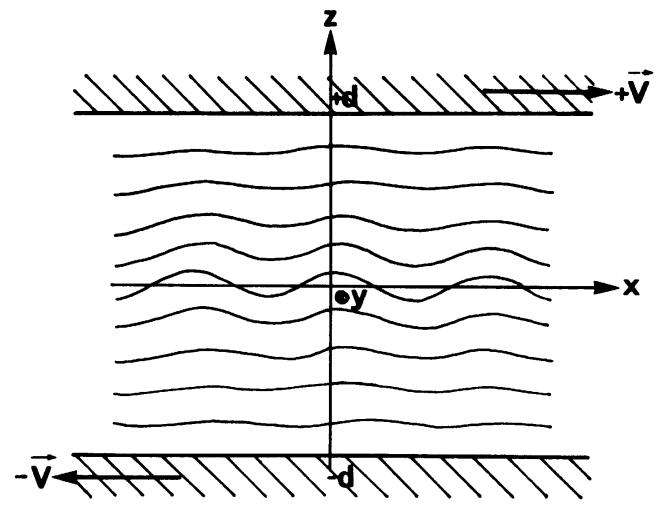

Fig. 1. - Sample geometry.

smectic layer elasticity. Equation (1) is valid under the assumptions that all viscosities can be replaced by a single coefficient $\eta$, that the mass density $\rho$ is constant and thus the incompressibility condition can be written as :

$$
\operatorname{div} \mathbf{V}=0 .
$$

Let $u$ be the displacement of the layers in the $z$-direction; then the elastic free energy density $F$ can be written as [6] : 


$$
F=\frac{1}{2} B\left\{\frac{\partial u}{\partial z}-\frac{1}{2}\left[\left(\frac{\partial u}{\partial x}\right)^{2}+\left(\frac{\partial u}{\partial y}\right)^{2}\right]\right\}^{2}+\frac{1}{2} K\left(\frac{\partial^{2} u}{\partial x^{2}}+\frac{\partial^{2} u}{\partial y^{2}}\right)^{2}
$$

where $B$ is the bulk modulus and $K$ is the splay modulus. The force density $G$ is the negative functional derivative of the energy density with respect to the displacement and can be explicitly written as :

$$
\begin{aligned}
G=-\frac{\delta F}{\delta u}=B \frac{\partial^{2} u}{\partial z^{2}}-K\left(\frac{\partial^{4} u}{\partial x^{4}}+2\right. & \left.\frac{\partial^{4} u}{\partial x^{2} \partial y^{2}}+\frac{\partial^{4} u}{\partial y^{4}}\right) \\
& -B\left[\frac{\partial u}{\partial z}\left(\frac{\partial^{2} u}{\partial x^{2}}+\frac{\partial^{2} u}{\partial y^{2}}\right)+2\left(\frac{\partial u}{\partial x} \frac{\partial^{2} u}{\partial x \partial z}+\frac{\partial u}{\partial y} \frac{\partial^{2} u}{\partial y \cdot \partial z}\right)-\frac{3}{2}\left(\frac{\partial u}{\partial x}\right)^{2} \frac{\partial^{2} u}{\partial x^{2}}\right. \\
& \left.-\frac{3}{2}\left(\frac{\partial u}{\partial y}\right)^{2} \frac{\partial^{2} u}{\partial y^{2}}-\frac{1}{2}\left(\frac{\partial u}{\partial x}\right)^{2} \frac{\partial^{2} u}{\partial y^{2}}-\frac{1}{2}\left(\frac{\partial u}{\partial y}\right)^{2} \frac{\partial^{2} u}{\partial x^{2}}-2 \frac{\partial u}{\partial x} \frac{\partial u}{\partial y} \frac{\partial^{2} u}{\partial x}\right] .
\end{aligned}
$$

The flow is coupled to the layer displacement by the permeation equation which, for an isothermal process, is :

$$
\frac{\mathrm{d} u}{\mathrm{~d} t}-V_{z}=\lambda_{\mathrm{p}} G
$$

where $\lambda_{\mathrm{p}}$ is the permeability [5].

Thermal effects will be neglected throughout.

For stationary shear without dilation equation (1) has the obvious solution :

$$
\begin{aligned}
\mathbf{V} & =\left(V_{0} z / d, 0,0\right) \\
u & =0
\end{aligned}
$$

where $V_{0}$ is the speed of the plates.

Now consider a sample that, while being sheared, is suddenly dilated by an amount $2 \delta$. If the dilation $\alpha=\delta / d$ is big enough the undulation instability develops.

In order to study this instability, we write the layer displacement in the form of a Fourier expansion :

$$
u=\alpha z+f(z) \exp [i(\mathbf{q} \cdot \mathbf{r}-\omega t)]
$$

which represents a homogeneous dilation with a superposed undulation of the layers having a frequency $\omega$ and a wave vector $\mathbf{q}=(q \cos \theta, q \sin \theta, 0)$ in the $(x y)$ plane inclined at an angle $\theta$ to the direction of shear $x$.

The threshold corresponds precisely to the point where the imaginary part of $\omega$ changes sign from negative to positive. Thus we may assume that the undulation does not decay and take $\omega$ real. We also assume that the undulation is not attenuated and take $q$ real as well. The phase velocity of the undulation is $c=\omega / q$. A detailed discussion of these aspects will be published elsewhere [8]. In what follows, the Fourier terms will be treated as a small perturbation. We write the velocity field as :

$$
\begin{aligned}
& V_{x}=\frac{V_{0} z}{d}+v_{x}=V+v_{x} \\
& V_{y}=v_{y} \\
& V_{z}=v_{z}
\end{aligned}
$$

where $\mathrm{v}=\left(v_{x}, v_{y}, v_{z}\right)$ is a non-stationary perturbation. Then, equation (1) can be rewritten as :

$$
\begin{aligned}
& \rho\left[\frac{\partial v_{x}}{\partial t}+V \frac{\partial v_{x}}{\partial x}+\frac{\mathrm{d} V}{\mathrm{~d} z} v_{z}\right]=\eta \nabla^{2} v_{x}-\frac{\partial P}{\partial x} \\
& \rho\left[\frac{\partial v_{y}}{\partial t}+V \frac{\partial v_{y}}{\partial x}\right] \quad=\eta \nabla^{2} v_{y}-\frac{\partial P}{\partial y} \\
& \rho\left[\frac{\partial v_{z}}{\partial t}+V \frac{\partial v_{z}}{\partial x}\right] \quad=\eta \nabla^{2} v_{z}-\frac{\partial P}{\partial z}+G
\end{aligned}
$$

The incompressibility condition becomes :

$$
\operatorname{div} \mathbf{v}=0 .
$$

The solution has to satisfy the boundary conditions :

$$
\mathbf{v}( \pm d)=0 \quad f( \pm d)=0 .
$$

We may calculate $G$ and $v_{z}$ from the equations (3)-(5). Linearizing and omitting the exponential factors we obtain :

$$
\begin{aligned}
& G=B\left(\frac{\mathrm{d}^{2} f}{\mathrm{~d} z^{2}}+\alpha q^{2} f\right)-k q^{4} f \\
& v_{z}=i q f(V \cos \theta-c)-\lambda_{\mathrm{p}} G .
\end{aligned}
$$

We wish to find an ordinary differential equation for the function $f(z)$.

A general calculation is too cumbersome to be useful. Yet, the mathematical problem can be substantially simplified while preserving its physical content by making the following assumptions :

(A.1) All functions vary much slower along $z$ than along $x$ or $y$, or equivalently, the wave length of the undulation is small compared to the sample thickness.

(A.2) Permeation can be neglected.

Permeation may, however, be important near the plates [9, 10]. A quantitative criterion for negligible permeation is :

$$
\eta \lambda_{\mathrm{p}} q^{2} \ll 1
$$


which is always satisfied. These assumptions will here be justified $a$ posteriori. They are discussed in another paper [8].

From equations $(9 a, b)$ we eliminate the pressure terms by taking cross derivatives. We substitute $v_{z}$ from (13) into the incompressibility condition (10) to obtain two equations which we solve for $v_{x}$ and $v_{y}$ :

$$
\begin{aligned}
& v_{x}=\cos \theta\left[c \frac{\mathrm{d} f}{\mathrm{~d} z}-\cos \theta \frac{\mathrm{d}}{\mathrm{d} z}(V f)\right] \\
& v_{y}=\sin \theta\left[c \frac{\mathrm{d} f}{\mathrm{~d} z}-\cos \theta \frac{\mathrm{d}}{\mathrm{d} z}(V f)\right] .
\end{aligned}
$$

Now we can eliminate the pressure from equations $(9 b, c)$ to find :

$$
G-i \eta q^{3}(V \cos \theta-c) f=0
$$

or, with (12), more explicitly :

$$
\frac{\mathrm{d}^{2} f}{\mathrm{~d} z^{2}}-\left[\lambda^{2} q^{4}+i T q^{3}\left(\frac{z}{d} V_{0} \cos \theta-c\right)-\alpha q^{2}\right] f=0
$$

where $\lambda=(K / B)^{1 / 2} \sim 10^{-7} \mathrm{~cm}$ is a characteristic length and $T=\eta / B \sim 10^{-1} \mathrm{~s}$ is a characteristic relaxation time.

We shall endeavor to solve it in the following sections.

2. Integral relation. - Before solving the fundamental equation (18) we shall rewrite it in an integral form which will allow us to see some interesting features.

We multiply (18) by $f^{*}$, the complex conjugate of $f$, and integrate the product across the sample thickness. Since, according to (11), $f$ vanishes at the boundaries, integration by parts yields, for the real and imaginary parts respectively, the relations :

$$
\begin{gathered}
\alpha=\frac{1}{q^{2}} \frac{\int_{-d}^{+d}|\mathrm{~d} f / \mathrm{d} z|^{2} \mathrm{~d} z}{\int_{-d}^{+d}|f|^{2} \mathrm{~d} z}+\lambda^{2} q^{2} \\
\int_{-d}^{+d}(V \cos \theta-c)|f|^{2} \mathrm{~d} z=0 .
\end{gathered}
$$

The right hand side of (19) is manifestly positive, whence it follows that the applied strain must be dilative $(\alpha>0)$ to bring about the undulation instability with, as well as without shear.

Relation (19) implies that there must be at least one level $z_{0}$ within the sample where the phase velocity equals the $x$-component of the flow velocity :

$$
z_{0} \in[-d,+d]: c=\frac{z_{0}}{d} V_{0} \cos \theta .
$$

Consider the "transversal " case $\theta=\pi / 2$ which corresponds to an undulation with a wave vector perpendicular to the direction of the shear $\mathbf{q}=(0, q, 0)$.

The velocity fields is now given by the trivial solution (6), thus $\mathbf{v}=0$. The fundamental equation reduces to :

$$
G=0
$$

which is simply the static equilibrium condition. The solution is well known [1] :

$$
f=\cos k z
$$

where

$$
k=\frac{\pi}{2 d} .
$$

Equation (19) becomes trivial and yields for the wave vector $q$ the equation :

$$
\alpha=k^{2} / q^{2}+\lambda^{2} q^{2} .
$$

Hence the threshold values for the dilation $\alpha$ and the wave vector $q$ become, respectively :

and

$$
\alpha_{\mathrm{c}}=\pi \lambda / d
$$

$$
q_{\mathrm{c}}=\sqrt{k / \lambda} \text {. }
$$

Equation (20) trivially implies the vanishing of the phase velocity $c=0$. That means that the undulation does not propagate. This is a non trivial result which we will prove to be generally valid.

We are now ready to justify our simplifying assumptions (A.1) and (A.2). Typical orders of magnitude for the quantities involved are : $2 d=100 \mu \mathrm{m}$, $\lambda=10^{-7} \mathrm{~cm}, \eta=1$ poise, $\lambda_{\mathrm{p}}=10^{-14} \mathrm{~g}^{-1} \mathrm{~cm}^{3} \mathrm{~s}$, hence $k \sim 300 \mathrm{~cm}^{-1}$ and $q_{\mathrm{c}} \sim 5 \times 10^{4} \mathrm{~cm}^{-1}$. Consequently, $k \ll q$, thus (A.1) is satisfied. Also

$$
\lambda_{\mathrm{p}} \eta q^{2} \sim 10^{-5} \ll 1,
$$

thus (A.2) is valid by an enormous margin.

3. Solution of the fundamental equation. - The fundamental equation (18) can, in principle, be formally solved in terms of Airy functions or, in a slightly more general case, in terms of parabolic cylinder functions. These, however involve complex parameters and variables and seem to be useless for a physical interpretation. Besides, in view of the approximations already made, the virtues of an "exact» solution are rather doubtful. Therefore we prefer to adopt a different approach and treat the shear perturbatively. More specifically, we assume that the speed $V_{0}$ is sufficiently small so that the term containing the product $z$.f in the fundamental equation (18) can be treated as a small perturbation. As a quantitative criterion we find immediately on inspection of equation (18) the strong inequality

$$
V_{0} \ll \frac{\lambda^{2} q}{T}=\frac{K q}{\eta}
$$


and substituting for $q$ its statical threshold value (27) :

$$
V_{0} \ll \frac{K}{\eta \sqrt{\lambda d}}=\eta^{-1} K^{3 / 4} B^{1 / 4} d^{-1 / 2} .
$$

With the quoted values of the constants we find a limiting speed of the order of $5 \times 10^{-2} \mathrm{~cm} / \mathrm{s}$. Under normal experimental conditions, when a undulation pattern can be observed, (29) is satisfied.

We now return to the fundamental equation (18). Quite generally, we may assume that its solution takes the form :

$$
f(z)=f_{0}[\cos k z+i \phi(z)]
$$

where $f_{0}$ is a constant length and $\phi(z)$ is the new unknown function to be determined. Substitution of (30) into (18) yields for $\phi$ the equation

$$
\frac{\mathrm{d}^{2} \phi}{\mathrm{d} z^{2}}+k^{2} \phi=T q^{3}\left(\frac{z}{d} V_{0} \cos \theta-c\right) \cos k z \text {. }
$$

Let us introduce the dimensionless quantities :

$$
\begin{gathered}
Z=k z=\frac{\pi z}{2 d}, \\
\Phi=\left(\frac{k}{q}\right)^{3}\left(\frac{d}{V_{0} T}\right) \phi=\left(\frac{\pi}{2 q}\right)^{3} \frac{B}{\eta V_{0} d^{2}} \phi
\end{gathered}
$$

and

$$
C=\frac{\pi c}{2 V_{0}} .
$$

Then (31) can be rewritten as

$$
\frac{\mathrm{d}^{2} \Phi}{\mathrm{d} Z^{2}}+\Phi=(Z \cos \theta-C) \cos Z .
$$

Its general solution is

$$
\begin{aligned}
& \Phi=A \cos Z+B \sin Z-\frac{1}{2} C Z \sin Z+ \\
& +\frac{1}{4} \cos \theta\left[Z \cos Z-\left(\frac{\pi}{2}-Z\right)\left(\frac{\pi}{2}+Z\right) \sin Z\right] .
\end{aligned}
$$

From the boundary conditions

$$
\Phi\left(Z= \pm \frac{\pi}{2}\right)=0
$$

it follows that

$$
B=0
$$

and

$$
C=0 \text {. }
$$

The fundamental equation (18) implies that the real and imaginary parts of $f$ must have opposite parities. Consequently, $\Phi$ must be an odd function of $Z$. Therefore, we find once more (39) and also :

$$
A=0 \text {. }
$$

The vanishing of $C$ is equivalent to the vanishing of the phase velocity $c$ and hence the vanishing of the frequency :

$$
\omega=0 \text {. }
$$

It is noteworthy that the orientation angle $\theta$ did not enter our considerations at all.

From basic symmetry arguments we have thus unambiguously shown that, at threshold, the undulation does not propagate; it remains stationary, irrespective of the mutual orientation between its wave vector $\mathbf{q}$ and the direction of the flow.

We are now ready to write down the explicit solution to the fundamental equation (18) :

$$
\begin{aligned}
f(Z) / f_{0} & =\cos Z+i \phi(Z) \\
& =\cos Z+i \varepsilon\left\{Z \cos Z-\left[\left(\frac{\pi}{2}\right)^{2}-Z^{2}\right] \sin Z\right\}
\end{aligned}
$$

where all multiplicative constants have been absorbed into the small parameter $\varepsilon$ (typically $\varepsilon \sim 10^{-3}$ ). The imaginary part is clearly a small correction. This justifies our perturbation approach. Finally, restoring dimensional variables, we have :

$$
\begin{aligned}
f(z)= & f_{0}\left\{\cos k z+i \frac{2}{\pi} q^{3} \frac{V_{0} T}{k^{2}} \cos \theta \times\right. \\
& \left.\times\left[k z \cos k z-\left(\frac{\pi^{2}}{4}-k^{2} z^{2}\right) \sin k z\right]\right\} .
\end{aligned}
$$

This result has a simple physical meaning. The imaginary part of $f(z)$ represents a perturbation of the sinusoidal wave form of the undulation, expressed in terms of a phase shift $\psi(z)$. Straightforward substitution into the real part of (7) yields for the layer displacement :

$$
u=\alpha z+f_{0} \cos k z \cos (\mathbf{q r}+\psi(z)) .
$$

The phase shift is explicitly:

$\psi(z)=\frac{2}{\pi} q^{3} \frac{V_{0} T \cos \theta}{k^{2}}\left\{k z-\left[\left(\frac{\pi}{2}\right)^{2}-(k z)^{2}\right] \tan k z\right\}$.

Note that $\psi$ is proportional to the velocity component $V_{0} \cos \theta$ and vanishes for $\theta=\pi / 2$.

To calculate the dilation $\alpha$, we substitute $f(z)$ from (43) into the integral relation (19) and obtain :

$$
\alpha=k^{2} / q^{2}+\lambda^{2} q^{2}+I\left(\frac{V_{0} T}{k} \cos \theta\right)^{2} q^{4}
$$


where $I$ is a numerical constant given by

$$
\begin{aligned}
I & =\frac{1}{\pi^{3}} \int_{-\pi / 2}^{+\pi / 2} \mathrm{~d} Z\left\{\left[\left(\frac{\pi}{2}\right)^{2} Z-Z^{3}\right] \sin Z-2 Z^{2} \cos Z\right\} \\
& =\frac{5}{4 \pi^{3}}-\frac{1}{12} \approx 0.0430 .
\end{aligned}
$$

In order to establish the threshold values we minimize (46) with respect to $q$.

A simple perturbative calculation yields :

$$
\alpha_{c}=2 k \lambda\left(1+I \frac{\left(V_{0} T \cos \theta\right)}{2 k \lambda^{3}}\right)^{2}
$$

for the threshold dilation, and

$$
q_{\mathrm{c}}=\sqrt{\frac{k}{\lambda}}\left(1-I \frac{\left(V_{0} T \cos \theta\right)^{2}}{2 k \lambda^{3}}\right)
$$

for the threshold wave vector.

Although the actual numerical values of the correction are negligible, these results are of great physical interest. The threshold dilation increases with the square of the velocity compount and has a minimum equal to the static value for $\theta=\pi / 2$. Thus the rotational symmetry in the plane is broken and, consequently, the first undulation to appear must be transversal, i.e., it must have a wave vector perpendicular to the flow. This agrees with what is observed.

Likewise, the threshold wave vector has a $(V \cos \theta)^{2}$ dependence, but now it is a maximum in the transversal direction $\theta=\pi / 2$.

4. Conclusion - There are two important conclusions to be drawn from the foregoing considerations.

At moderate shear rates, i.e. as long as some undulation pattern can be established, the undulation stays stationary. This is a novel result which is by no means a priori obvious. It is valid at least in the approximation defined by the assumptions (A.1,2) and (28) which cover the range of physical interest.

Whereas in the static case all directions parallel to the layers are equivalent, even a slight shear makes the transversal direction highly privileged. It has the lowest threshold dilation, equal to the static value which thus becomes an absolute threshold. Moreover, it is the only direction in which the undulation behaves precisely like a static one. This result is rigorous, since the coupling between the undulation and the shear flow exactly vanishes for transversal shear.

When, at some higher dilation, a two dimensional undulation develops, its second principal direction must necessarily be perpendicular to the first, hence it will be parallel to the shear flow. Therefore the pattern will be rectangular and well aligned throughout the sample and will thus have the aspect of a two dimensional "crystal».

This contrasts with a sample subjected to dilation in the absence of shear where undulations nucleate at many places and a disordered "glassy" pattern will be established.

Thus the most important effect of an applied shear flow is the breaking of the rotational symmetry of the smectic sample.

Quantitatively we find, that the threshold values are hardly affected at all.

Experimentally one observes in smectic A liquid crystals, at high dilation values, two dimensional undulations which have already developed into defect patterns, specifically networks of focal parabolas [11]. Clearly, these patterns inherit the symmetry at threshold. Without shear, the patterns have a " glassy " aspect [12]. However, even a weak shear applied prior to the dilation will bring about alignment along and across the shear direction, while even at moderately high shear rates the patterns always stay stationary.

There is perfect agreement between our theory and experiment.

Acknowledgments. - The authors are deeply indebted to Maurice Kléman for many stimulating and illuminating discussions. One of us (SIBA) wishes to express his sincere gratitude to the D.G.R.S.T. for a grant and the Laboratoire de Physique des Solides for the hospitality which made possible his participation in this research.

\section{References}

[1] Clark, N. A., Meyer, R. B., Appl. Phys. Lett. 22 (1973) 493.

[2] Delaye, M., Ribotta, R., Durand, G., Phys. Lett. 44 (1973) 139.

[3] Delrieu, J. M., J. Chem. Phys. 60 (1974) 1081.

[4] Ribotta, R., Durand, G., J. Physique 38 (1977) 179.

[5] Martin, P., Parodi, O., Pershan, P., Phys. Rev. A 6 (1972) 2401.

[6] De Gennes, P. G., The Physics of Liquid Crystals (Oxford Univ. Press) 1975.
[7] Helfrich, W., Phys. Rev. Lett. 23 (1969) 372.

[8] Ben Abraham, S. I., Oswald, P., Submitted to publication to Mol. Cryst. Liq. Cryst.

[9] De Gennes, P. G., Phys. Fluids 17 (1974) 1645.

[10] Rapini, A., J. Physique-Lett. 37 (1976) L-49.

[11] Oswald, P., Béhar, J., Kléman, M., submitted to publication to Philos. Mag.

[12] Rosenblatt, Ch. S., Pindak, R., Clark, N. A., Meyer, R. B., J. Physique 38 (1977) 1105. 